\title{
The Cooper Pair Electrons Origination in Copper Oxide
}

\section{Superconductors}

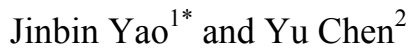 \\ 1. School of Physics and Telecommunication Engineering, Shaanxi University of Technology, Hanzhong 723001, China \\ 2. School of Chemistry and Environmental Science, Shaanxi University of Technology, Hanzhong 723001, China
}

\begin{abstract}
An unambiguous determination of the pairing electrons origination in copper oxide superconductors in necessary to understanding the origin of high-temperature superconductivity. Several years ago, several experiments have produced strong evidence for d-wave pairing symmetry. This result also rules out different from the symmetry in conventional superconductors. Using quantum chemical bond theory to research into the effect of chemical bonding on the feature of element nature change and its resulting change on the copper oxide superconductivity. The origin of Cooper pair electrons in the copper oxide is studied with the symmetry characteristics of superconducting electrons. A conclusion is obtained that Cooper pairs of high $\mathrm{T}_{\mathrm{c}}$ copper oxide originate the no-bonded electrons of the bearing superconducting elements. This is an important notion of a thorough understanding of mechanism of high $T_{c}$ copper oxide superconducting.
\end{abstract}

Key words: High $\mathrm{T}_{\mathrm{c}}$ copper oxide, Cooper pair electron origin, non-bond electrons, quantum chemical bond theory.

\section{Introduction}

In 1986, Bednorz and Muller discovered that La-Ba-Cu-O chemical compounds have high superconducting transition temperature [1]. In 1987, C.E. Gough. et al. [2], confirmed that high $\mathrm{T}_{\mathrm{c}}$ Copper oxide superconductor is based on Cooper pairing (i.e., the frequency of Shapiro steps is $2 \mathrm{eV} / \mathrm{h}$ ). And the characteristics of Cooper pairing are also proved by a series of experiments, like DC (direct current) and AC (alternating current) Josephson effect [3], Andreev reflection current [4] and electron-hole mixture resolution in photoemission spectroscopy [5]. Electron bounding mechanism of high $T_{c}$ copper oxide superconductors evolves into a key to probing into mechanism of superconductivity. All the elements in high $T_{c}$ copper oxide superconductors are formed by chemical bonding causing great changes in the element nature, which, in turn, exerts great impact on the features of superconductivity. Quantum chemical bond

Corresponding author: Jinbin Yao, professor, research fields: the high $\mathrm{T}_{\mathrm{c}}$ superconducting mechanism and theoretical physics. E-mail: yjinbini@126.com. theory is a crucial theory origin from the application of quantum mechanics to chemical bonding among elements [6]. And quantum chemical bonding has already been successful applied to the researches into elements bonding and matter nature in such fields as chemistry, biology and material science.

Quantum chemical bond theory is mainly concerned with the Pauling quantum chemical bonding theory [6] and Sanderson electronegativity equalization principle [7], with the former (Pauling) directed at researches into the characteristics when the elements formation chemical bond and the latter (Sanderson) at researches into interrelations with the electronegativity equalization after the elements formation.

\section{Methods}

In light of the characteristics of the high $\mathrm{T}_{\mathrm{c}}$ copper oxide superconductors' superconductivity and properties of superconductors, the $\mu_{\mathrm{B}}$ ( $\mu_{\mathrm{B}}$ is Coulomb's interaction between the bonded electrons) of the crystal lattice stability is correlated with the bonded electrons to satisfy the condition of $\mu_{\mathrm{B}}>\lambda_{\mathrm{B}}\left(\lambda_{\mathrm{B}}\right.$ is the coupling constant of the bonded electrons and phonons) while $\lambda_{C}$ 
( $\lambda_{\mathrm{C}}$ is the coupling constant of formation Cooper pairs electrons and phonons) is correlated with non-bonded electrons to satisfy the condition of $\lambda_{\mathrm{C}}>\mu_{\mathrm{C}}\left(\mu_{\mathrm{C}}\right.$ is Coulomb's interaction between the Cooper pairs electrons) [8]. Due to the chemical bond formation of the elements in the high $T_{c}$ copper oxide superconductors, the $\mathrm{N}(\mathrm{O})(\mathrm{N}(\mathrm{O})$ is a kind of the density of the Bloch of one spin per unity energy at the Fermi surface $)$ and $V\left(V=\left\langle V_{K K}\right\rangle_{\text {arerage, }}\right.$ the matrix element and $V_{K K}$, can be replaced by a constant average matrix element, for pairs making transition in the region $-\hbar \omega<\varepsilon<\hbar \omega$ and by zero outside this region, where $\omega$ is the average phonon frequency) will have special change, and the electron media phonon can generate high $\mathrm{T}_{\mathrm{c}}$ superconductivity [8].

As $\mathrm{Cu}$ and $\mathrm{O}$ on the $\mathrm{CuO}_{2}$ plane of the high $\mathrm{T}_{\mathrm{c}}$ copper oxide superconductors have obvious isotope effects and they are inter-correlated, the phonons of $\mathrm{Cu}$ and $\mathrm{O}$ on $\mathrm{CuO}_{2}$ plane participate in the electrons phonons coupling [9].

Because of chemical bond formation of $\mathrm{Cu}$ and $\mathrm{O}$ on $\mathrm{CuO}_{2}$ plane, the $f_{\mathrm{Cu}}=f_{\mathrm{O}}\left(f_{\mathrm{Cu}}\right.$ is the force of the bonded electrons exerted with $\mathrm{Cu}$ element while $f_{\mathrm{O}}$ is the force of the bonded electrons exerted with $\mathrm{O}$ element) is obtained. The bonded electrons are not beneficial to the superconductivity. The coupling electrons of $\mathrm{Cu}$ and $\mathrm{O}$ on $\mathrm{CuO}_{2}$ plane are the locality of non-bonded electrons [9].

\section{Results and Discussion}

Pauling gave the definition of the chemical bonds[6] that as far as two atoms or atomic groups were concerned, if the force that acted between them could lead to the formation of aggregation and the stability of the aggregation was big enough for chemists to treat it as an independent category of element, it was claimed that there were chemical bonds between these atoms or atom groups and in the chemical bonds there were ionic bonds and covalent bonds.

Pauling maintained that [6] the ionicity of the bonds was caused by the difference in the abilities of attracting electrons of atoms in molecules. When the chemical compounds of $\mathrm{AB}$ type was formed and the abilities of attracting electrons of atom $\mathrm{B}$ was bigger than those of atom $\mathrm{A}$ and even big enough to make them form the type of $\mathrm{A}^{+} \mathrm{B}^{-}$, this bond was the ideal ionic bond. When the abilities of attracting electrons of atom $\mathrm{B}$ was not enough to form the type of $\mathrm{A}^{+} \mathrm{B}^{-}$but the electrons of the bond a little deviated to atom $\mathrm{B}$, the ionic bonds were partially formed.

Because of the formation of the ionic bonds, there were great changes in the nature of the formed ionic bonds.

The changes of the formation of $\mathrm{A}^{+}$were listed as follows:

(1) The number of the outermost electrons of atom $\mathrm{A}^{+}$was much more than that of atom $\mathrm{A}$;

(2) The structure of the outermost electrons of Atom $\mathrm{A}^{+}$was different from that of Atom $\mathrm{A}$ and the formed magnetism of atom $\mathrm{A}^{+}$was completely different from that of atom $\mathrm{A}$;

(3) The radius of atom $\mathrm{A}^{+}$was smaller than that of atom $\mathrm{A}$;

(4) The outermost electronic ionic potential of atom $\mathrm{A}^{+}$was far bigger than that of atom $\mathrm{A}$.

The changes of the formation of $\mathrm{B}^{-}$were listed as follows:

(1) The number of the outermost bonded electrons of atom $\mathrm{B}^{-}$was far less than that of atom $\mathrm{B}$;

(2) Because of the electronic structure formed by the bonded electrons, the outermost of atom $\mathrm{B}^{-}$differed from that of Atom B;

(3) The radius of Atom $\mathrm{B}^{-}$was far bigger than that of atom $\mathrm{B}$;

(4) The ionic potential of the outermost bonded electrons of atom $\mathrm{B}^{-}$was much less than that of atom $\mathrm{B}$.

According to the relationship among $\mathrm{N}\left(\mathrm{E}_{\mathrm{F}}\right)$, the number of the electrons and the radius of Fermi surface in Ref. [8] of the references, the relationship between $\mathrm{V}$ and ionic potential could be obtained as follows. In the ionic bonds, the bonded electrons could not form the effective Cooper pair to Atom $\mathrm{A}^{+}$, Atom $\mathrm{B}^{-}$or atom $\mathrm{A}^{+\delta q}$ or atom $\mathrm{B}^{+\delta q}$, alone produce high $\mathrm{T}_{\mathrm{c}}$. 
Other magnetic variations and changes of radius could produce direct influence on the replacement of the doped crystal lattice and the magnetism and the lattice constant of the replaced chemical compound.

Fig. 1 shows the relaxation rate under $\mathrm{T}_{\mathrm{c}}$ in TI-2223 [10] and Fig. 2 shows the temperature change of relaxation in some typical substances[11]. Compared with the about artificial superlattices ${ }^{63} \mathrm{Cu}$ relaxation rate Fig. 1a in superconductor $\mathrm{Nb} / \mathrm{Cu}$, two features about high $\mathrm{T}_{\mathrm{c}}$ copper oxide superconductors become prominent: (1) no coherent peak exists around $\mathrm{T}_{\mathrm{c}}\left(\mathrm{T}<\mathrm{T}_{\mathrm{c}}\right)$; (2) When $\mathrm{T}$ $<\mathrm{T}_{\mathrm{c}}$, relaxation rate takes on a power temperature changes instead of index temperature changes. In other words, copper oxidant is not s-wave superconductor.

Another key experiment which convinces researchers that copper oxide is not s-wave superconductor is the one that relaxation rate and frequency shift are affected by non-magnetic impurities and crystalline imperfections. In BCS superconductors, magnetic impurities may destroy Cooper pairs and reduce $T_{c}$ and the scattering of non-magnetic impurities can increase the energy gap isotropy. However, it is this scattering that exerts great changes on d-wave superconductor properties which have anisotropic energy gap.

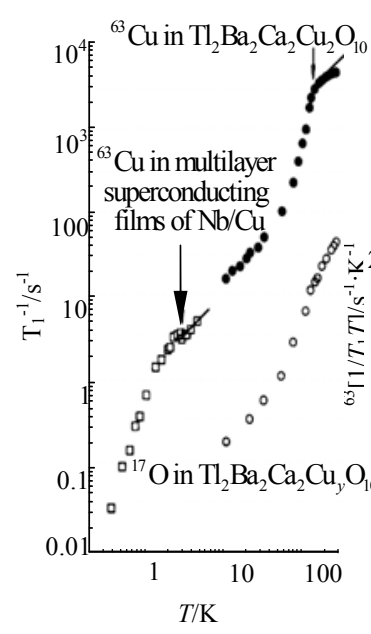

(a)

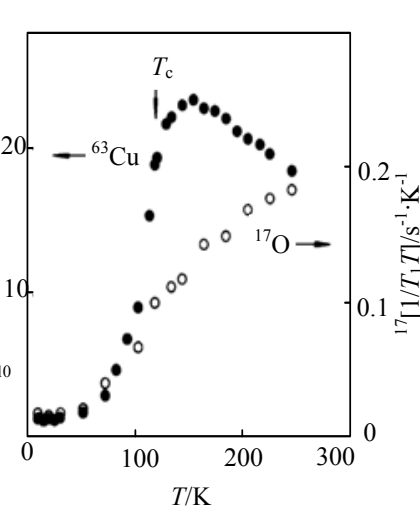

(b)
Fig. 1 The properties of (a) the relaxation of $\mathrm{Cu}$ and $\mathrm{O}$ in Tl-2223 (arrow for $T_{c}$, line for the $T_{1} T=$ constant) and (b) the changes of the Tl-2223 with temperature.
When d-wave is scattering singly, the biggest characteristic is that low-energy excited states change and finite density of states will occur in the vicinity of $\mathrm{E}=\mathrm{E}_{\mathrm{F}}$ which is called residual density of states Nres [12], as is shown in Fig. 3.

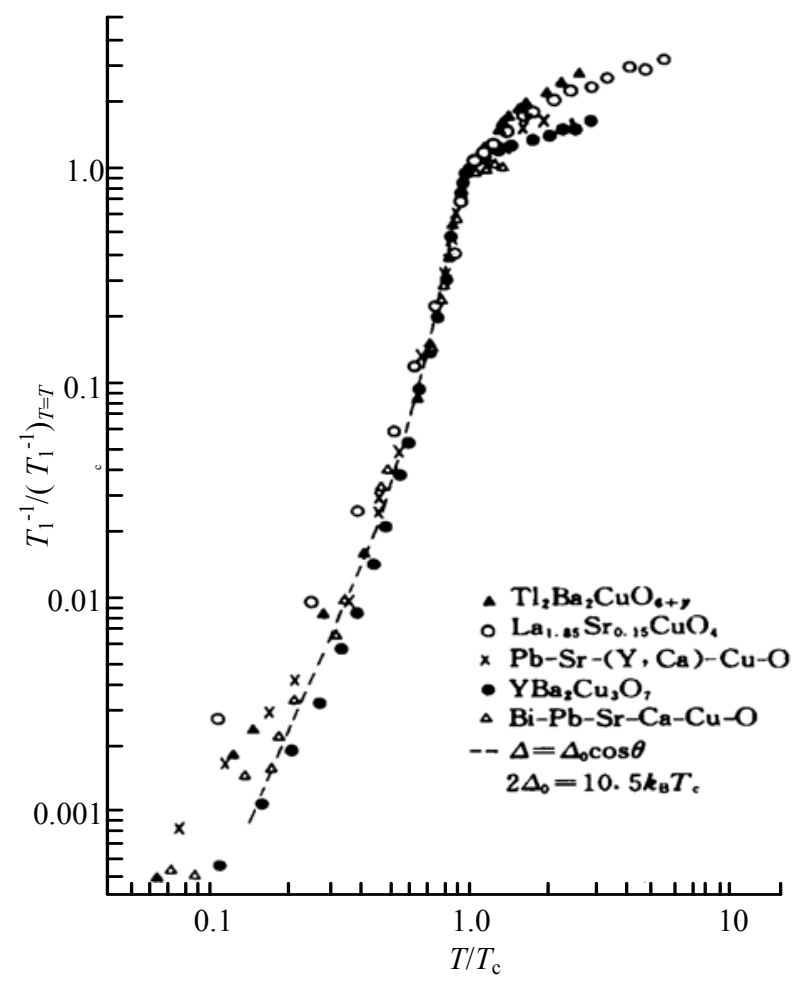

Fig. 2 Superconducting state $T_{1}$ of various materials. They all basically fall on the same curve.

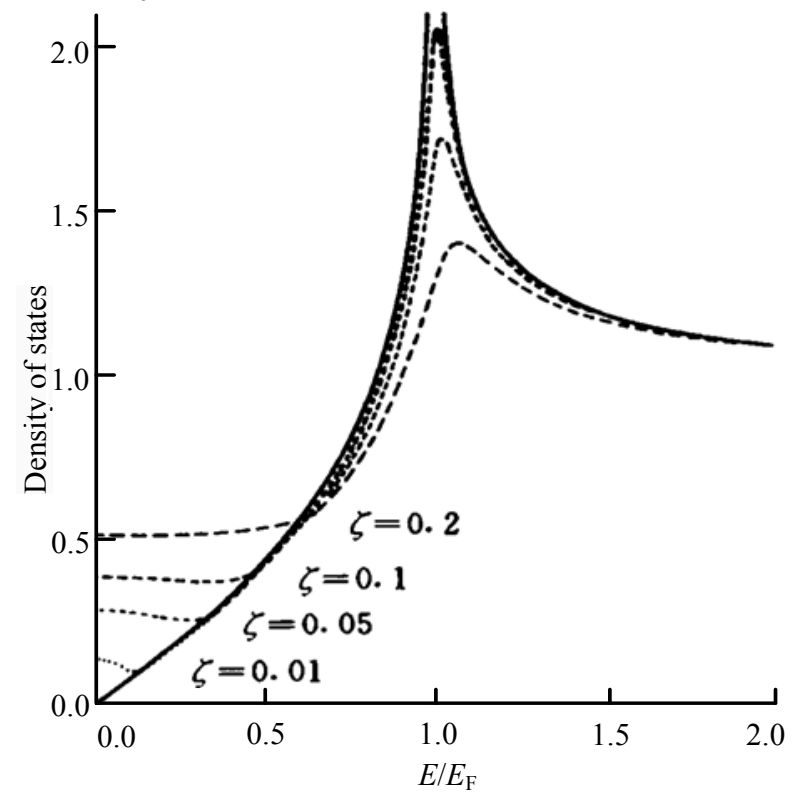

Fig. $3 d_{x^{2}-y^{2}}$ superconducting state density change with impurities, in figure, the $\zeta$ for doping degree. 


\section{Conclusions}

We use the quantum chemical bond theory to study the Cooper pair electrons origination in copper oxide superconductors. A conclusion has obtained that the Cooper pair electrons of high $\mathrm{T}_{\mathrm{c}}$ copper oxide originate from non-bonded electron of the elements with carrying superconducting.

\section{References}

[1] Bednorz, J., and Muller, K. A. 1986. "Possible High $T_{\mathrm{c}}$ Superconductivity in the Ba-La-Cu-O System." Z. Phys. B 64: 189-193.

[2] Gough, C. E., Colclough, M. S., Forgan, E. M., Jordan, R. G., Keene, M., Muirhead, C. M., and et al. 1987. "Flux-Quantization in a High- $T_{c}$ Superconductor." Nature 326: 855.

[3] Yamashita, T., Kawakami, A., Nishihara, T., Hirotsu, Y., and Takata, M. 1987. "AC Josephson Effect in Point-Contacts of Ba-Y-Cu-O Ceramics." Jpn. J. Appl. Phys. 26: L635.

[4] Hoevers, H. F. C., van Bentum, P. J. M., van de Leemput, L. E. C., van Kempen, H., Schellingerhout, A. J. G., and van der Marel, D. 1988. "Determination of the Energy Gap in a Thin $\mathrm{YBa}_{2} \mathrm{Cu}_{3} \mathrm{O}_{7-\mathrm{x}}$ Film by Andreev Reflection and by Tunneling." Physica C 152: 105-110.
[5] Campuzano, J. C., Ding, H., Norman, M. R., Randeira, M., Bellman, A. F., Mochiku, T., and et al. 1996. "Direct Observation of Particle-Hole Mixing in the Superconducting State by Angle-Resolved Photoemission." Phys. Rev. B 53: 14737-14740.

[6] Pauling, L. 1996. The Nature of Chemical Bond and the Structure of Molecules and Crystals. Cornell University Press.

[7] Sanderson, R. T. 1951. "An Interpretation of Bond Lengths and a Classification of Bonds." Science 114: 670-2.

[8] Chen, Y., and Huang, X. M. 2010. "N(O) Distribution and Electrons Distribution of Atomic Nucleus Outside, Correlation the between the V and Ionic Potential." Journal of Low Temperature Physics 32: 303-7. (in Chinese)

[9] Chen, Y., Huang, X. M., and Wang, J. W. 2010. "Origination of the High $T_{\mathrm{c}}$ Superconductivity of Copper Oxides from Electrons Media Phonons." Journal of materials Science and Engineering 4: 67-75.

[10] Zheng, G. Q., Kitaoka, Y., Asayama, K., Hamada, K., Yamauchi, H., and Tanaka, S. 1995. "Characteristics of the Spin Fluctuation in $\mathrm{Tl}_{2} \mathrm{Ba}_{2} \mathrm{Ca}_{2} \mathrm{Cu}_{3} \mathrm{O}_{10}$." J. Phys. Soc. Jpn. 64: 3184-7.

[11] Asayama, K., Zheng, G. Q., Kitaoka, Y., Ishida, K., and Fujiwara, K. 1991. "NMR Study of High-T Superconductors." Physica C 178: 281-6.

[12] Hotta, T. 1993. "Impurity Effects in Cuprate Superconductors.” J. Phys. Soc. Jpn. 62: 274-280. 